\title{
Lactate-related Risk Score (the LaRi Score): A robust risk scoring system of hepatocarcinoma patients' prognosis base on lactate-related genes
}

\author{
Zijian ZHANG ${ }^{* 1,2}$, Jian XU², Yuyuan $\mathrm{CHEN}^{2}$, Qi ZHOU \\ 'Digestive Medicine Center, The Seventh Affiliated Hospital of Sun Yat-Sen University, Shenzhen, China \\ ${ }^{2}$ Department of General Surgery, The Seventh Affiliated Hospital of Sun Yat-Sen University, Shenzhen, China \\ ${ }^{3}$ Department of General Surgery, Huiya Hosiptal of The First Affiliated Hospital of Sun Yat-Sen University, Huizhou, China
}

Introduction: Hepatocarcinoma (HCC) is one of the most lethal cancers worldwide. Lactate has recently been proved as a novel role in both metabolism and epigenetic in cancers. Our study aims to establish and validate a novel scoring system based on lactate-related genes to estimate the prognosis of HCC patients.

Methods: Our study established a robust model to predict HCC patients' prognosis. Data from TCGA, ICGC, and GEO were explored. A LASSO-SVM-RFE algorithm model, consensus clustering, and GDSC drug prediction were used to choose prognostic genes/ subtypes and potential drugs.

Results: We establish a 4-mRNA robust model that can distinguish high-risk patients from low-risk patients with different prognosis significantly. The AUC of the Kaplan-Meier curve in 1 year, 3 years, and 5 years are all indicating the good performance of the LaRi Score. Moreover, Gene Ontology (GO) enrichment analysis results showed that several epigenetic regulation pathways and targets' expressions are significantly associated with high LaRi Score, which means lactate-related genes could participate in tumorigenesis not only in metabolic but also epigenetic regulation. And the potential drugs are shown by the mean of GDSC drug predictions.

Conclusions: In conclusion, the LaRi Score provides a novel method to evaluate the prognosis of HCC patients based on the lactate-related genes. According to the GO enrichment results and GDSC drug-sensitive predictions, new therapeutic targets or drugs focus on the lactate metabolism and epigenetic function will be discovered and developed for HCC patients. 